\title{
Risk of being convicted of theft and other crimes in anorexia nervosa and bulimia nervosa: A prospective cohort study in a Swedish female population
}

\author{
Shuyang Yao, MSc ${ }^{1}$ (D) | Ralf Kuja-Halkola, PhD' | Laura M. Thornton, PhD2 | \\ Claes Norring, PhD 3,4 | Catarina Almqvist, MD, PhD ${ }^{1,5}$ | Brian M. D'Onofrio, PhD ${ }^{6}$ | \\ Paul Lichtenstein, PhD1 ｜ Niklas Långström, MD, PhD1,7 | Cynthia M. Bulik, PhD1,2,8 | \\ Henrik Larsson, $\mathrm{PhD}^{1,9}$
}

${ }^{1}$ Department of Medical Epidemiology and Biostatistics, Karolinska Institutet, Stockholm, Sweden

${ }^{2}$ Department of Psychiatry, University of North Carolina at Chapel Hill, Chapel Hill, North Carolina

${ }^{3}$ Department of Clinical Neuroscience, Karolinska Institutet, Stockholm, Sweden

${ }^{4}$ Stockholm Center for Eating Disorders, Stockholm Health Care Services, Stockholm, Sweden

${ }^{5}$ Astrid Lindgren Children's Hospital, Karolinska University Hospital, Stockholm, Sweden

${ }^{6}$ Department of Psychological and Brain Science, Indiana University, Bloomington, Indiana

${ }^{7}$ Department of Neuroscience, Uppsala University, Uppsala, Sweden

${ }^{8}$ Department of Nutrition, University of North Carolina at Chapel Hill, Chapel Hill, North Carolina

${ }^{9}$ School of Medical Sciences, Örebro University, Örebro, Sweden

\section{Correspondence}

Shuyang Yao, Department of Medical Epidemiology and Biostatistics, Karolinska Institutet, Nobels väg 12A, Stockholm, Sweden.

Email: shuyang.yao@ki.se

\section{Funding information}

China Scholarship Council (S.Y.); Swedish

Research Council (C.M.B.), Grant Number: VR Dnr: 538-2013-8864; Swedish Research Council, Grant Number: 2011-3060; Swedish Initiative for Research on Microdata in the Social and Medical Sciences (SIMSAM) framework, Grant Number: 340-2013-5867.

\begin{abstract}
Objective: We examined epidemiological associations between anorexia nervosa (AN) and bulimia nervosa $(\mathrm{BN})$ and risks of committing theft and other crimes in a nationwide female population.

Method: Females born in Sweden during 1979-1998 $(N=957,106)$ were followed from age 15 for up to 20 years using information on clinically diagnosed AN and BN (exposures), convictions of theft and other crimes (outcomes), psychiatric comorbidities, and familial relatedness from Swedish national registers. We estimated hazard ratios (HRs) of criminality in exposed versus unexposed females using Cox proportional hazards regressions and explored how comorbidities and unmeasured familial factors explained the associations.

Results: The cumulative incidence of convictions of theft (primarily petty theft) and other crimes was higher in exposed females (AN: $11.60 \%$ theft, 7.39\% other convictions; BN: $17.97 \%$ theft, $13.17 \%$ other convictions) than in unexposed females ( $\sim 5 \%$ theft, $\sim 6 \%$ other convictions). The significantly increased risk of being convicted of theft in exposed females (AN: HR $=2.51,95 \%$ confidence interval $=[2.29,2.74], \mathrm{BN}: 4.31[3.68,5.05]$ ) was partially explained by comorbidities; unmeasured familial factors partially explained the association with convictions of theft in BN but not in AN. Females with BN had a doubled risk of convictions of other crimes, which was partially explained by comorbidities.

Discussion: Individuals with eating disorders had increased risk for convictions of theft and potentially other crimes. Results underscore the importance of regular forensic screening and encourage research on mechanisms underlying the relation between crime and eating disorder psychopathology and efforts to determine how best to address such relation in treatment.
\end{abstract}

\section{KEYWORDS}

anorexia nervosa, bulimia nervosa, cohort study, crime, eating disorders, epidemiology, registerbased, stealing, theft behavior 


\section{1 | INTRODUCTION}

Eating disorders, including anorexia nervosa (AN) and bulimia nervosa (BN), are psychiatric disorders that have broad impact on health and quality of life in afflicted individuals (Fichter \& Quadflieg, 2016; Smink, van Hoeken, \& Hoek, 2013; Whiteford et al., 2013). Criminality, among other adversities (Herzog, Nussbaum, \& Marmor, 1996; Smink et al., 2013; Yao et al., 2016), has been associated with eating disorders (Coker, Smith, Westphal, Zonana, \& McKee, 2014). As it might add to the psychosocial burden on individuals who are already suffering from the direct effects of eating disorders (Grant \& Kim, 2002, 2005), it is important to verify the association between eating disorders and criminal behaviors and to understand mechanisms of the associations. However, research on criminality in eating disorders is quite limited. The majority of previous reports came from clinical observations focusing on theft behavior in eating disorders (Baum \& Goldner, 1995; Crisp, Hsu, \& Harding, 1980; McElroy, Hudson, Pope, \& Keck, 1991; Mitchell, Fletcher, Gibeau, Pyle, \& Eckert, 1992). Rates of committing theft were typically greater in individuals with $B N$ and the binge-purge subtype of AN than in individuals with the restrictive subtype of AN (McElroy, Pope, Hudson, Keck, \& White, 1991; Vandereycken \& van Houdenhove, 1996), and have been related to increased levels of impulsivity, comorbidities, and severity of eating disorders (Herzog et al., 1996; Krahn, Nairn, Gosnell, \& Drewnowski, 1991). Only one study was population-based (Coker et al., 2014), but relied on selfratings from a US adolescent sample, and reported increased risks of committing theft and other crimes in individuals with binge eating behaviors compared with individuals without (Coker et al., 2014).

Psychiatric morbidity such as personality disorders and attentiondeficit hyperactivity disorder (ADHD)-both associated with increased risk of crime (Coker et al., 2014; Vinkers, de Beurs, Barendregt, Rinne, \& Hoek, 2011)-co-occur with eating disorders and could account for any observed associations between eating disorders and crime (Hudson, Hiripi, Pope, \& Kessler, 2007). Twin and family studies have established underlying genetic and familial environmental liabilities for both eating disorders and criminality (Rhee \& Waldman, 2002; Yilmaz, Hardaway, \& Bulik, 2015), such liabilities might overlap and confound the associations. Alternative explanations to the associations between eating disorders and criminality have not been previously explored.

We conducted a prospective cohort study using registry data from a nationally representative female cohort in Sweden to thoroughly examine the associations between clinically diagnosed $A N$ and $B N$ and convictions of theft and other crimes. We considerably expand the literature not only by relying on population-based registers for identification of both exposures (clinically diagnosed $\mathrm{AN}$ and $\mathrm{BN}$ ) and outcomes (convictions of theft and other crimes), but also by carefully attending potential confounders that have not been considered in previous research. Interlinked Swedish population-based registers provided diagnostic information on eating disorders and comorbid psychiatric disorders and criminal conviction records, and the large population size allowed us to account for their potential impact on observed associations. In addition to a standard cohort design (i.e., population-level comparison where we compared the risks of outcomes in differentially exposed unrelated individuals in the study population), we employed a sibling-comparison design in which we compared the risk of crime in differentially exposed full-sisters (i.e., sisters born to the same biological parents) to adjust for unmeasured familial factors; defined as factors shared by full-sisters such as some genetic and familial environmental factors (D'Onofrio, Lahey, Turkheimer, \& Lichtenstein, 2013).

\section{2 | METHOD}

This study was approved by the Regional Ethics Review Board in Stockholm, Sweden. The requirement for informed consent was waived because the study was based on administrative population-based registers. An independent government agency (Statistics Sweden) merged and de-identified register data and delivered them for research purposes (Ludvigsson et al., 2016).

\section{1 | Data sources}

With the unique individual identification number in registry we linked several national registers. The Swedish Total Population Register provides information on birth year and month, death date, and migration type and date (Ludvigsson et al., 2016). The National Patient Register (NPR) contains inpatient psychiatric diagnoses since 1973 and outpatient psychiatric diagnoses since 2001; diagnoses in the NPR are based on the Swedish versions of the International Classification of Diseases, Eighth Revision (ICD-8, 1973-1986), ICD-9 (1987-1996), and ICD-10 (since 1997) (Ludvigsson et al., 2011). Two quality registers for eating disorders, that is, Swedish National Quality Register for Eating Disorder Treatment (Riksät, since 1999) and quality assurance system for eating disorders (Stepwise, since 2005), provide eating disorder diagnoses from specialized treatment centers across Sweden (Birgegard, Bjorck, \& Clinton, 2010; Emilsson, Lindahl, Koster, Lambe, \& Ludvigsson, 2015), with increased coverage over time (Javaras et al., 2015). Quality register diagnoses are based on the Diagnostic and Statistical Manual of Mental Disorders, 4th Edition, Text Revision (DSM-IV-TR) (American Psychiatric Association, 2000). The Criminal Conviction Register (held by the National Council for Crime Prevention) provides criminal conviction records from Swedish lower courts since 1973, regardless of the medico-legal disposition of the convicted offender (Frisell, Pawitan, Langstrom, \& Lichtenstein, 2012). Since the age of criminal responsibility in Sweden is 15 years (Frisell, Pawitan, et al., 2012), law-breaking behaviors before age 15 years are not registered. The Multi-Generation Register (Statistics Sweden) links biological parents to individuals who were born after 1932 and lived in Sweden any time after 1961. Information from these registers was updated through December 31, 2013.

\section{2 | Study population}

The study population included females born in Sweden between January 1, 1979 and December 31, 1998. Adoptees, those whose biological parents were not identifiable in the Multi-Generation Register, and those 
who died or emigrated before age 15 years were excluded, yielding a total study population of 957,106 females. They were followed from age 15 years to the time when they left the cohort, that is, when the participant (a) experienced the outcome, (b) emigrated, (c) died, or (d) reached December 31, 2013, whichever came first. We further linked females with the same biological parents using parents' personal identification numbers in the Multi-Generation Register, to identify full-sisters in the study population.

\subsection{Exposures: AN and BN}

The two exposures (not mutually exclusive) were $\mathrm{AN}$ and $\mathrm{BN}$ diagnosed before or during follow-up. The exposure AN included clinically diagnosed AN or atypical AN, identified with ICD-9 code 307B or ICD10 codes F50.0 or F50.1 or meeting DSM-IV-TR criteria for AN or Eating Disorder Not Otherwise Specified (EDNOS) examples 1 and 2. The exposure $\mathrm{BN}$ included clinically diagnosed $\mathrm{BN}$ or atypical $\mathrm{BN}$, identified with ICD-10 codes F50.2 or F50.3 or meeting DSM-IV-TR criteria for BN or EDNOS example 3. We obtained diagnoses from the NPR and the quality registers for eating disorders. The period for diagnosing $\mathrm{BN}$ was shorter than that for $\mathrm{AN}$, as $\mathrm{BN}$ was not an independent eating disorder category in the Swedish version of ICD-9 ("Klassifikation av Sjukdomar 1987," 1987) but was identifiable in ICD-10 (since 1997), potentially leading to more under-detection of $B N$ cases than of $A N$ cases.

The exposures are time-varying. Females whose AN or BN was first diagnosed before or at age 15 years were treated as exposed during the entire follow-up. Females whose AN or BN was first diagnosed after age 15 years but before leaving the cohort were treated as unexposed from age 15 years to the date of the diagnosis and as exposed afterwards.

\subsection{Outcomes: Convictions of theft and other crimes}

We defined the primary outcome as convictions of theft, identified in the Criminal Conviction Register and defined it as the first conviction according to the Swedish Penal Code Chapter 8 Sections 1, 2, 4, 7-11, and 13 (i.e., theft, petty theft, gross theft, vehicle theft, unlawful dispossession, self-repossession, unlawful diversion of energy, unlawful takes from a forest or field if not considered as trespassing, and theft committed against a person living with or closely related to the convicted person). We also explored being convicted of "other crimes"; defined as the first nontheft conviction. The outcome date was defined as the date of committing the crime, if registered; otherwise, we used the date of conviction as the outcome date.

\section{5 | Comorbidities}

We included other eating disorders, personality disorders, and ADHD diagnosed at any time as covariates, as they have been associated with both eating disorders and criminality in a way that we believe could confound the associations of interest (Bulik et al., 2010; Coker et al., 2014; Hudson et al., 2007; Vinkers et al., 2011). Other eating disorders were defined as having any eating disorder diagnosis other than the exposure (identified by ICD-9 codes 307B or $307 \mathrm{~F}$ or ICD-10 codes F50.0, F50.1, F50.2, F50.3, or F50.9 or meeting DSM-IV-TR criteria for an eating disorder, except the exposure). Personality disorders were identified with ICD-9 code 301 or ICD-10 codes F60-F61. ADHD was defined as ICD-9 code 314 or ICD-10 code F90 or being prescribed with specific ADHD medication(s) as defined in previous studies (Chen et al., 2017).

\section{6 | Statistical analysis}

To illustrate the unadjusted associations between the exposures and outcomes, we plotted the estimated cumulative incidence of being convicted of theft and other crimes in exposed and unexposed groups using the Kaplan-Meier method (software: R 3.2.2). Note that given the time-varying feature of the exposures, the estimated cumulative incidence curve could be interpreted as a proportion of individuals with outcome event among individuals who did not change their exposure status during follow-up, if the association between current exposure status and outcomes were independent of previous exposure status (Lichtenstein et al., 2012). We also estimated the overall incidence rates in exposed and unexposed females and unadjusted incidence rate ratios during follow-up.

Next, we performed population-level comparisons to estimate the relative risks of being convicted of theft and other crimes in females exposed to $\mathrm{AN}$ and $\mathrm{BN}$ compared to unexposed, unrelated females in the study population. Cox proportional hazards regressions were applied (Model 1) to estimate hazard ratio (HR) as a measure of relative risk. To account for the potential effect of comorbidities on the associations, we further adjusted for other eating disorders, personality disorders, and ADHD (Model 2). To explore the effect of each of the comorbidities, we adjusted for them separately in sensitivity analyses. To account for the potential effect of unmeasured familial factors, we conducted sibling-comparisons, where stratified Cox proportional hazards regressions were applied to estimate HRs of being convicted of theft and other crimes in females exposed to $\mathrm{AN}$ and $\mathrm{BN}$ compared to their unexposed full-sisters in the study population (Model 3). By comparing the risks of outcomes in differentially exposed full-sisters, sibling-comparisons automatically controlled for unmeasured familial factors shared by full-sisters in a family. Contrasting estimates from population-level comparisons versus sibling-comparisons could inform whether unmeasured familial factors influenced tested associations. Simplistically, if unmeasured familial factors mainly accounted for the associations, we would expect decreased effect size of association estimates in sibling-comparisons compared with population-level comparisons. Conversely, if the associations were independent of unmeasured familial factors, we would expect similar estimates in both populationlevel and sibling-comparisons. Note that only females with differently exposed full-sisters were included in Model 3.

All the Cox models used attained age as underlying time scale and were adjusted for birth year as a categorical variable. We addressed nonindependency of data using a cluster-robust estimator of standard error and visually examined Schoenfeld residuals to verify the validity 
TABLE 1 Model-related description of the study population

\begin{tabular}{|c|c|c|c|c|}
\hline & Exposed to AN & Unexposed to $\mathrm{AN}$ & Exposed to $\mathrm{BN}$ & Unexposed to $\mathrm{BN}$ \\
\hline \multicolumn{5}{|l|}{ Theft as outcome } \\
\hline Population in each group, $N^{a}$ & 11,114 & 954,581 & 5,197 & 957,007 \\
\hline Outcome events, $\mathrm{N}$ & 496 & 39,940 & 159 & 40,278 \\
\hline Follow-up time, in person-year & $64,629.27$ & $8,767,634.87$ & $26,278.95$ & $8,805,985.19$ \\
\hline $\begin{array}{l}\text { Incidence rate, cases per } 1000 \\
\text { person-years }(95 \% \mathrm{Cl})\end{array}$ & $7.67(7.03,8.38)$ & $4.56(4.51,4.60)$ & $6.05(5.18,7.07)$ & $4.57(4.53,4.62)$ \\
\hline Incidence rate ratio $(95 \% \mathrm{Cl})$ & $1.59(1.46,1.74)$ & Ref & $1.40(1.20,1.64)$ & Ref \\
\hline \multicolumn{5}{|l|}{ Other crimes as outcome } \\
\hline Population in each group, $N^{a}$ & 11,245 & 954,581 & 5,394 & 957,007 \\
\hline Outcome events, $N$ & 316 & 41,376 & 168 & 41,524 \\
\hline Follow-up time, in person-year & $66,992.91$ & $8,810,309.54$ & $27,607.49$ & $8,849,694.97$ \\
\hline $\begin{array}{l}\text { Incidence rate, cases per } 1000 \\
\text { person-years }(95 \% \mathrm{Cl})\end{array}$ & $4.72(4.22,5.27)$ & $4.70(4.65,4.74)$ & $6.09(5.23,7.08)$ & $4.69(4.65,4.74)$ \\
\hline Incidence rate ratio $(95 \% \mathrm{Cl})$ & $0.95(0.85,1.06)$ & Ref & $1.33(1.14,1.55)$ & Ref \\
\hline
\end{tabular}

Note. $\mathrm{AN}=$ anorexia nervosa; $\mathrm{BN}=$ bulimia nervosa; $\mathrm{N}=$ number of observations; $95 \% \mathrm{Cl}=95 \%$ confidence interval; Ref $=$ reference group for calculating incidence rate ratio.

aparticipants could first be unexposed and then became exposed; therefore, the sum of exposed and unexposed populations was greater than the total population.

of the proportional hazards assumption. We performed data management in SAS 9.4 and analyses in Stata 13.0.

\section{3 | RESULTS}

\section{1 | Descriptive statistics}

The 957,106 females in the study population were from 736,538 families. We identified 410,026 biological full-sisters from 189,458 families. Descriptive statistics are presented in Table 1. Convictions of both theft and other crimes were more prevalent in females exposed to eating disorders than in unexposed females (except other crimes in AN) and more prevalent in females exposed to $\mathrm{BN}$ than in females exposed to AN (Figure 1). After up-to-20-year follow-up since age 15 years, the estimated cumulative incidence for conviction of theft (i.e., the estimated probability of being convicted of theft before age 35) was $11.60 \%$ (95\% confidence interval [95\% Cl], 10.53\%-12.77\%) in females exposed to AN and $17.97 \%(14.19 \%-22.62 \%)$ in females exposed to BN, compared to $5.04 \%(4.98 \%-5.09 \%)$ and $5.07 \%(5.01 \%-5.12 \%)$ in females unexposed to $\mathrm{AN}$ and $\mathrm{BN}$, respectively. The distribution of subtypes of theft did not differ significantly between exposed and unexposed females for both AN and BN, with "petty theft" being the most common subtype (Supporting Information Table S1). The corresponding numbers for being convicted of other crimes were $7.39 \%$ $(6.47 \%-8.43 \%)$ in females exposed to $\mathrm{AN}$ and $13.17 \%(10.79 \%-$ $16.04 \%)$ in females exposed to BN, compared to $6.24 \%(6.16 \%-6.32 \%)$ and $6.23 \%(6.16 \%-6.31 \%)$ in females unexposed to $\mathrm{AN}$ and $\mathrm{BN}$, respectively.

\section{2 | Hazard ratios of being convicted of theft and other crimes in eating disorders}

HRs of being convicted of theft in eating disorders are presented in Table 2. Population-level comparisons (Model 1) revealed that the risk of being convicted of theft in females exposed to AN was more than twice the risk of unexposed females (HR [95\% Cl]: 2.51 [2.29, 2.74]). The increased risk of being convicted of theft in AN was partially explained by other eating disorders, personality disorders, and ADHD (1.84 [1.67, 2.02], Model 2), but remained similar in sibling-comparison (3.31 [2.57, 4.28], Model 3), suggesting unmeasured familial factors were not likely to explain the association. We observed a stronger association with convictions of theft in $\mathrm{BN}$ than in $\mathrm{AN}$; the risk of being convicted of theft in females exposed to $\mathrm{BN}$ was more than four times the risk in unexposed females (4.31 [3.68, 5.05], Model 1). HRs of being convicted of theft in $\mathrm{BN}$ decreased when adjusting for psychiatric comorbidities (2.62 [2.23, 3.09], Model 2) and in sibling-comparison (2.78 [1.69, 4.60], Model 3), suggesting that comorbidities and unmeasured familial factors partially explained the association between $\mathrm{BN}$ and the convictions of theft.

Table 3 presents the HRs of being convicted of other crimes in eating disorders. Population-level comparisons (Model 1) revealed a weak association between $\mathrm{AN}$ and being convicted of other crimes (1.16 $[1.04,1.29])$, which disappeared when adjusting for psychiatric comorbidities (Model 2) or unmeasured familial factors (Model 3). A stronger association with convictions of other crimes was found in BN (2.15 $[1.85,2.51]$, Model 1$)$, which attenuated but remained statistically significant after adjusting for psychiatric comorbidities (1.47 [1.26, 1.73], Model 2) and remained similar when adjusting for unmeasured familial 

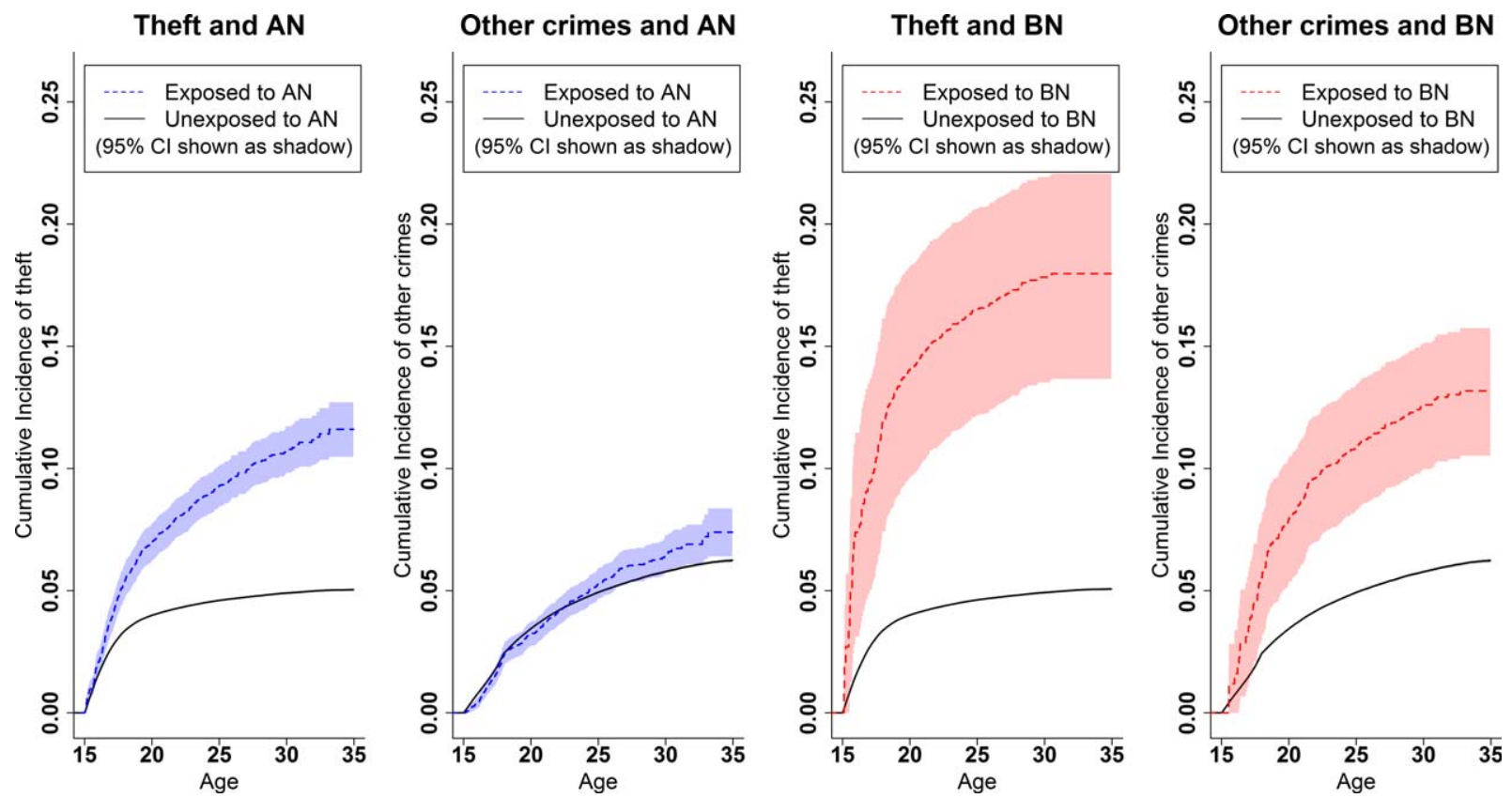

FIGURE 1 Cumulative incidence of being convicted of theft and other crimes in females exposed and unexposed to AN and BN. Note. Both theft and other crimes occurred more frequently in females exposed to eating disorders than in unexposed females (except other crimes in AN), and more frequently in females exposed to BN than in females exposed to AN. After up-to-20-year follow-up since age 15 years, the estimated cumulative incidence of being convicted of theft (i.e., the estimated probability of being convicted of theft before age 35 ) was $11.60 \%$ in females exposed to $\mathrm{AN}$ and $17.97 \%$ in females exposed to BN, compared to $5.04 \%$ and $5.07 \%$ in females unexposed to AN and BN, respectively. The corresponding numbers for other crimes were $7.39 \%$ in females exposed to AN and $13.17 \%$ in females exposed to BN, compared to $6.24 \%$ and $6.23 \%$ in females unexposed to $\mathrm{AN}$ and $\mathrm{BN}$, respectively. [Color figure can be viewed at wileyonlinelibrary.com]

factors in sibling-comparison (1.91 [1.23, 2.95], Model 3). The effect of each comorbid psychiatric disorder on the examined associations is presented in Supporting Information (Supporting Information Table S2).

\section{4 | DISCUSSION}

In this large cohort study, we thoroughly explored the associations between $\mathrm{AN}$ and $\mathrm{BN}$ and the risk of being convicted of theft and other crimes in a Swedish female population, while attending to the potential influences of comorbidities and unmeasured familial factors (i.e., factors shared by full-sisters including genetic factors and various shared environmental factors such as familial lifestyle factors). We found increased risk of being convicted of theft in females exposed to $\mathrm{AN}$ or $\mathrm{BN}$ compared to unexposed females. BN was also associated with increased risk of being convicted of other types of crime. Both absolute and relative risks were higher in $\mathrm{BN}$ than in $\mathrm{AN}$. The increased risk of criminality in eating disorders is worthy of clinical attention as convictions could increase stress and anxiety, interrupt treatment, and adversely influence the course of treatment and recovery (Goldschmidt et al., 2014).

\section{$4.1 \mid$ Theft}

The large size of the cohort provided sufficient statistical power to control for several important psychiatric comorbidities: other eating

TABLE 2 The relative risks of being convicted of theft in eating disorders in population-level and sibling-comparisons

\begin{tabular}{|c|c|c|c|c|c|c|}
\hline \multirow[b]{2}{*}{ Theft } & \multicolumn{3}{|l|}{ AN } & \multicolumn{3}{|l|}{$\underline{B N}$} \\
\hline & $\mathrm{HR}(95 \% \mathrm{Cl})$ & $p$-value & $\begin{array}{l}\text { No. informative } \\
\text { individuals (families) }\end{array}$ & $\mathrm{HR}(95 \% \mathrm{CI})$ & $p$-value & $\begin{array}{l}\text { No. informative } \\
\text { individuals (families) }\end{array}$ \\
\hline Model 1 & $2.51(2.29,2.74)$ & $<.001$ & $957,105(736,537)$ & $4.31(3.68,5.05)$ & $<.001$ & $957,106(736,538)$ \\
\hline Model 2 & $1.84(1.67,2.02)$ & $<.001$ & $957,105(736,537)$ & $2.62(2.23,3.09)$ & $<.001$ & $957,106(736,538)$ \\
\hline Model 3 & $3.31(2.57,4.28)$ & $<.001$ & $975(419)^{a}$ & $2.78(1.69,4.60)$ & $<.001$ & $439(191)^{a}$ \\
\hline
\end{tabular}

Note. $\mathrm{AN}=$ anorexia nervosa; $\mathrm{BN}=$ bulimia nervosa; $\mathrm{HR}=$ hazard ratio; $95 \% \mathrm{Cl}=95 \%$ confidence interval. Model 1: Population-level comparison; Cox proportional hazards regression was applied, adjusted for birth year. Model 2: Population-level comparison; based on Model 1, Model 2 additionally adjusted for other eating disorders, personality disorders, and ADHD. Model 3: Sibling-comparison; stratified Cox proportional hazards regression was applied, adjusted for birth year. Compared with Model 1, Model 3 additionally adjusted for unmeasured familial factors shared by full-sisters. Estimates with $p$-value $<.05$ are presented as bold characters.

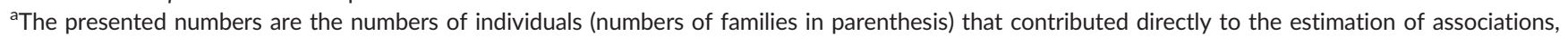
other individuals in the population might contribute indirectly to the estimation. 
TABLE 3 The relative risks of being convicted of other crimes in eating disorders in population-level and sibling-comparisons

\begin{tabular}{|c|c|c|c|c|c|c|}
\hline \multirow[b]{2}{*}{ Other crimes } & \multicolumn{3}{|l|}{ AN } & \multicolumn{3}{|l|}{$\mathrm{BN}$} \\
\hline & HR (95\% Cl) & $p$-value & $\begin{array}{l}\text { No. informative } \\
\text { individuals (families) }\end{array}$ & HR (95\% Cl) & $p$-value & $\begin{array}{l}\text { No. informative } \\
\text { individuals (families) }\end{array}$ \\
\hline Model 1 & $1.16(1.04,1.29)$ & .01 & $957,105(736,538)$ & $2.15(1.85,2.51)$ & $<.001$ & $957,105(736,538)$ \\
\hline Model 2 & $0.93(0.83,1.04)$ & .20 & $957,105(736,538)$ & $1.47(1.26,1.73)$ & $<.001$ & $957,105(736,538)$ \\
\hline Model 3 & $1.14(0.86,1.52)$ & .36 & $639(274)^{a}$ & $1.91(1.23,2.95)$ & .004 & $379(166)^{a}$ \\
\hline
\end{tabular}

Note. $\mathrm{AN}=$ anorexia nervosa; $\mathrm{BN}=$ bulimia nervosa; $\mathrm{HR}=$ hazard ratio; $95 \% \mathrm{Cl}=95 \%$ confidence interval. Model 1: Population-level comparison; Cox proportional hazards regression was applied, adjusted for birth year. Model 2: Population-level comparison; based on Model 1, Model 2 additionally adjusted for other eating disorders, personality disorders, and ADHD. Model 3: Sibling-comparison; stratified Cox proportional hazards regression was applied, adjusted for birth year. Compared with Model 1, Model 3 additionally adjusted for unmeasured familial factors shared by full-sisters. Estimates with $p$-value $<.05$ are presented as bold characters.

${ }^{a}$ The presented numbers are the numbers of individuals (numbers of families in parenthesis) that contributed directly to the estimation of associations, other individuals in the population might contribute indirectly to the estimation.

disorders, personality disorders, and ADHD (Hudson et al., 2007; Vinkers et al., 2011). Adjusting for the comorbidities decreased the associations with being convicted of theft in $\mathrm{AN}$ and $\mathrm{BN}$, suggesting that the associations were partially explained by the comorbidities. Comparing the results from population-level comparisons and siblingcomparisons informed whether unmeasured familial factors explained the associations. The association between $\mathrm{AN}$ and being convicted of theft remained similar in population-level comparison and in siblingcomparison, suggesting that the association may reflect direct effects between $\mathrm{AN}$ and being convicted of theft rather than confounding effects of unmeasured familial factors shared by full-sisters. It is nevertheless important to note that the association between $\mathrm{AN}$ and being convicted of theft could still be confounded by factors that were not shared by full-sisters, which were not captured or specified by our measurements and study design. Future studies with different designs could further explore specific factors that explain the association. In contrast, the association between $\mathrm{BN}$ and the risk of being convicted of theft was greater in population-level comparison than in siblingcomparison, suggesting that the increased risk of being convicted of theft in BN could be partially explained by unmeasured familial factors, such as genetic and shared familial environmental factors. We were unable to answer more detailed questions about the nature of the unmeasured familial factors for BN and committing theft, but suggest that impulsivity might be one important shared factor to consider in future research, as it is a heritable trait that has been associated with both BN and committing theft (Bezdjian, Baker, \& Tuvblad, 2011; Grant \& Kim, 2002; Wildes \& Marcus, 2013). Interestingly, AN and BN differed in the extent to which unmeasured familial factors explained their associations with theft, which may reflect differences in their etiologies.

\section{2 | Other crimes}

To our knowledge, only one prior study has explored associations between eating disorders and the risk of committing nontheft crimes and reported increased risk of committing other crimes in individuals with any binge eating behaviors based on self-report measures (Coker et al., 2014). Similarly, we observed increased risk of being convicted of other crimes in females with clinically diagnosed BN and further found that the association was partially explained by psychiatric comorbidities. However, the overall estimate of risk of being convicted of "other crimes," a broadly defined group, does not necessarily reflect how each type of crime is associated with eating disorders. More detailed investigations are needed to elucidate the precise nature of the associations between eating disorders and the risk of committing various types of nontheft crimes.

\subsection{Clinical implications}

The clinical implications of the results are three-fold. First, the associations should inform clinicians of the increased risk of criminality in individuals with eating disorders. Related legal issues may introduce stress that hampers recovery and increases the risk of relapse in individuals with eating disorders (Goldschmidt et al., 2014; Grilo et al., 2012). Second, criminal behavior might suggest the presence of multi-impulsive or emotionally dysregulated forms of eating disorders (Wildes \& Marcus, 2013). Individuals with this form of eating disorder may have other comorbid impulsive behaviors and poorer treatment response (Wildes \& Marcus, 2013). Finally, the results may suggest a more direct effect of eating disorders (especially $\mathrm{AN}$ ) on the risk of being convicted of theft, although this study was unable to explore specific mechanisms. Unfortunately, we were unable to obtain information on the motivation for theft or specifics on the items being stolen; studies that provide greater detail on such information may aid in understanding the nature of theft behaviors in individuals with eating disorders and how it differs from individuals without eating-related psychopathology who also engage in theft behaviors.

\section{4 | Limitations}

This study has several limitations. First, as register data captured only treatment-seeking individuals and since the coverage of registers has increased over time (Javaras et al., 2015), individuals with eating disorders who remained outside of the health care system would not be 
identifiable from registers, resulting in exposure misclassification. The misclassification of $\mathrm{BN}$ is likely to be greater than that of $\mathrm{AN}$ since $\mathrm{BN}$ was not a separate diagnosis in the Swedish version of ICD-9 while AN was ("Klassifikation av Sjukdomar 1987," 1987). This exposure misclassification is probably nondifferential in terms of the criminal outcomes that happened subsequent to diagnosis, and would most likely result in underestimations of the true effects (Flegal, Brownie, \& Haas, 1986). In addition, the fact that eating disorder exposures in this study reflected cases that were identified by the health care system, and knowing that a significant proportion of individuals with eating disorders do not seek medical treatment (Ali et al., 2017; Regan, Cachelin, \& Minnick, 2017), may limit the generalizability of the results within the more severe end of the diagnostic spectrum. Second, many criminal behaviors in Sweden are not reported, meaning that law-breaking individuals may not be charged or convicted (Victims' tendency to report crime, 2008), resulting in misclassification of criminal outcomes in our study. Such misclassification would bias the estimates of associations if it differed between exposed and unexposed individuals; otherwise, if independent of the eating disorder, the misclassification is not likely to bias the estimations of relative risks (such as HRs). The results were based on information on convictions (i.e., did not include crimes that were not reported or crimes that were committed but did not lead to convictions) and may have limited generalizability to any criminal behaviors. Third, siblingcomparisons account for unmeasured familial factors that make siblings similar, such as genetic factors, shared diet, familial socioeconomic conditions, and so forth. However, these factors may also have nonshared effects (e.g., full-siblings may cope differently with poor family socioeconomic conditions) and the sibling-comparisons are unable to adjust for such nonshared effects of common risk factors. Such residual confounders could even bias the estimates from siblingcomparisons if full-sisters are less similar in terms of these confounders than of exposures (Frisell, Oberg, Kuja-Halkola, \& Sjolander, 2012), but we were unable to quantify such influences or specify such factors. Fourth, prior knowledge could not inform whether comorbid psychiatric disorders were confounders, mediators, and/or colliders (i.e., common results of eating disorders and criminality) (Greenland, 2003) in terms of the associations of interest. Adjusting for comorbidities could introduce bias, if, for instance, the common causes of exposure and comorbidities are independent from the common causes of outcome and comorbidities (D'Onofrio et al., 2016; Greenland, 2003; Yao et al., 2016). Therefore, we did not adjust for more comorbidities and encourage caution when interpreting the adjusted results. Fifth, due to the low prevalence of $A N$ and $B N$ in males in our data, we were unable to conduct parallel analyses for boys and men. Therefore, our results may not generalize to males with eating disorders. Finally, given that the ICD codes could not distinguish between the restrictive and binge/purge subtypes of AN, we were unable to determine whether risk of criminality differed by AN subtype. Similarly, as binge-eating disorder is not specified as an independent diagnostic category in ICD 8, 9, or 10, we could not examine the association of the criminal outcomes with this eating disorder presentation.

\section{5 | CONCLUSION}

In this large population-based study, we observed increased risk of being convicted of theft and other crimes in AN and BN. The association with convictions of theft and other crimes was stronger in $\mathrm{BN}$ than in AN and was partially explained by comorbidities. BN, but not AN, might share common familial factors with theft behaviors. With reference to clinical practice, although taking a criminal/forensic history should indeed be a component of all psychiatric evaluations, in practice, this may not always be the case with eating disorders. The failure to assess forensic history may in part be due to (erroneous) preconceived notions related to the demographics of the majority of patients with eating disorders (i.e., adolescent to young adult females) as being a low-risk population for criminal activity. Our results provide an important reminder to clinicians to routinely screen for criminal/forensic history in patients with eating disorders.

\section{ACKNOWLEDGMENTS}

S. Yao acknowledges financial support from China Scholarship Council. Dr. Bulik acknowledges funding from the Swedish Research Council (VR Dnr: 538-2013-8864). Financial support was provided by the Swedish Research Council (Grant Number: 2011-3060) and through the Swedish Initiative for Research on Microdata in the Social And Medical Sciences (SIMSAM) framework, Grant Number: 340-2013-5867.

\section{CONFLICT OF INTEREST}

Dr. Norring is a consultant on a research grant from Shire. Dr. Lichtenstein has served as a speaker for Medice. Dr. Bulik is a consultant for and grant recipient from Shire. Dr. Larsson has served as a speaker for Eli-Lilly and Shire and has received research grants from Shire; all outside this study.

\section{REFERENCES}

Ali, K., Farrer, L., Fassnacht, D. B., Gulliver, A., Bauer, S., \& Griffiths, K. M. (2017). Perceived barriers and facilitators towards help-seeking for eating disorders: A systematic review. International Journal of Eating Disorders, 50(1), 9-21. doi:10.1002/eat.22598

American Psychiatric Association. (2000). Diagnostic and statistical manual of mental disorders: DSM-IV-TR. (4th ed., text revision. ed.). Washington, DC: American Psychiatric Association.

Baum, A., \& Goldner, E. M. (1995). The relationship between stealing and eating disorders: A review. Harvard Review of Psychiatry, 3(4), 210-221.

Bezdjian, S., Baker, L. A., \& Tuvblad, C. (2011). Genetic and environmental influences on impulsivity: A meta-analysis of twin, family and adoption studies. Clinical Psychological Review, 31(7), 1209-1223. doi:10.1016/j. cpr.2011.07.005

Birgegard, A., Bjorck, C., \& Clinton, D. (2010). Quality assurance of specialised treatment of eating disorders using large-scale Internet-based collection systems: Methods, results and lessons learned from designing the Stepwise database. European Eating Disorders Review, 18(4), 251-259. doi:10.1002/erv.1003

Bulik, C. M., Thornton, L. M., Root, T. L., Pisetsky, E. M., Lichtenstein, P., \& Pedersen, N. L. (2010). Understanding the relation between anorexia 
nervosa and bulimia nervosa in a Swedish national twin sample. Biological Psychiatry, 67(1), 71-77. doi:10.1016/j.biopsych.2009.08.010

Chen, Q., Brikell, I., Lichtenstein, P., Serlachius, E., Kuja-Halkola, R., Sandin, S., \& Larsson, H. (2017). Familial aggregation of attentiondeficit/hyperactivity disorder. Journal of Child Psychology Psychiatry, 58, 231-239. doi:10.1111/jcpp.12616

Coker, K. L., Smith, P. H., Westphal, A., Zonana, H. V., \& McKee, S. A. (2014). Crime and psychiatric disorders among youth in the US population: An analysis of the National Comorbidity Survey-Adolescent Supplement. Journal of American Academy of Child Adolescence Psychiatry, 53(8), 888-898.e2. doi:10.1016/j.jaac.2014.05.007

Crisp, A. H., Hsu, L. K., \& Harding, B. (1980). The starving hoarder and voracious spender: Stealing in anorexia nervosa. Journal of Psychosomatic Research, 24(5), 225-231.

D'Onofrio, B. M., Class, Q. A., Rickert, M. E., Sujan, A. C., Larsson, H., Kuja-Halkola, R., ... Oberg, A. S. (2016). Translational epidemiologic approaches to understanding the consequences of early-life exposures. Behavior Genetics, 46(3), 315-328. doi:10.1007/s10519-015-9769-8

D'Onofrio, B. M., Lahey, B. B., Turkheimer, E., \& Lichtenstein, P. (2013). Critical need for family-based, quasi-experimental designs in integrating genetic and social science research. American Journal of Public Health, 103(Suppl 1), S46-S55. doi:10.2105/AJPH.2013.301252

Emilsson, L., Lindahl, B., Koster, M., Lambe, M., \& Ludvigsson, J. F. (2015). Review of 103 Swedish healthcare quality registries. Journal of Internal Medicine, 277(1), 94-136. doi:10.1111/joim.12303

Fichter, M. M., \& Quadflieg, N. (2016). Mortality in eating disordersResults of a large prospective clinical longitudinal study. International Journal of Eating Disorders, 49(4), 391-401. doi:10.1002/eat.22501

Flegal, K. M., Brownie, C., \& Haas, J. D. (1986). The effects of exposure misclassification on estimates of relative risk. American Journal of Epidemiology, 123(4), 736-751.

Frisell, T., Oberg, S., Kuja-Halkola, R., \& Sjolander, A. (2012). Sibling comparison designs: Bias from non-shared confounders and measurement error. Epidemiology, 23(5), 713-720. doi:10.1097/EDE.0b013e31825fa230

Frisell, T., Pawitan, Y., Langstrom, N., \& Lichtenstein, P. (2012). Heritability, assortative mating and gender differences in violent crime: results from a total population sample using twin, adoption, and sibling models. Behavior Genetics, 42(1), 3-18. doi:10.1007/s10519-011-9483-0

Goldschmidt, A. B., Wonderlich, S. A., Crosby, R. D., Engel, S. G., Lavender, J. M., Peterson, C. B., ... Mitchell, J. E. (2014). Ecological momentary assessment of stressful events and negative affect in bulimia nervosa. Journal of Consulting Clinical Psychology, 82(1), 3039. doi:10.1037/a0034974

Grant, J. E., \& Kim, S. W. (2002). Clinical characteristics and associated psychopathology of 22 patients with kleptomania. Comprehensive Psychiatry, 43(5), 378-384.

Grant, J. E., \& Kim, S. W. (2005). Quality of life in kleptomania and pathological gambling. Comprehensive Psychiatry, 46(1), 34-37. doi: 10.1016/j.comppsych.2004.07.022

Greenland, S. (2003). Quantifying biases in causal models: classical confounding vs collider-stratification bias. Epidemiology (Cambridge, Mass.), 14(3), 300-306.

Grilo, C. M., Pagano, M. E., Stout, R. L., Markowitz, J. C., Ansell, E. B., Pinto, A., .. Skodol, A. E. (2012). Stressful life events predict eating disorder relapse following remission: Six-year prospective outcomes. International Journal of Eating Disorders, 45(2), 185-192. doi:10.1002/eat.20909

Herzog, D. B., Nussbaum, K. M., \& Marmor, A. K. (1996). Comorbidity and outcome in eating disorders. Psychiatry Clinical North America, 19 (4), 843-859.

Hudson, J. I., Hiripi, E., Pope, H. G., Jr., \& Kessler, R. C. (2007). The prevalence and correlates of eating disorders in the National Comorbidity
Survey Replication. Biological Psychiatry, 61(3), 348-358. doi: 10.1016/j.biopsych.2006.03.040

Javaras, K. N., Runfola, C. D., Thornton, L. M., Agerbo, E., Birgegard, A., Norring, C., ... Bulik, C. M. (2015). Sex- and age-specific incidence of healthcare-register-recorded eating disorders in the complete Swedish 1979-2001 birth cohort. International Journal of Eating Disorders, 48(8), 1070-1081. doi:10.1002/eat.22467

Krahn, D. D., Nairn, K., Gosnell, B. A., \& Drewnowski, A. (1991). Stealing in eating disordered patients. Journal of Clinical Psychiatry, 52(3), 112-115.

Lichtenstein, P., Halldner, L., Zetterqvist, J., Sjolander, A., Serlachius, E., Fazel, S., ... Larsson, H. (2012). Medication for attention deficithyperactivity disorder and criminality. New England Journal of Medicine, 367(21), 2006-2014. doi:10.1056/NEJMoa1203241

Ludvigsson, J. F., Almqvist, C., Bonamy, A. K., Ljung, R., Michaelsson, K., Neovius, M., ... Ye, W. (2016). Registers of the Swedish total population and their use in medical research. European Journal of Epidemiology, 31(2), 125-136. doi:10.1007/s10654-016-0117-y

Ludvigsson, J. F., Andersson, E., Ekbom, A., Feychting, M., Kim, J. L., Reuterwall, C., ... Olausson, P. O. (2011). External review and validation of the Swedish national inpatient register. BMC Public Health, 11, 450. doi:10.1186/1471-2458-11-450

McElroy, S. L., Hudson, J. I., Pope, H. G., \& Keck, P. E. (1991). Kleptomania: Clinical characteristics and associated psychopathology. Psychological Medicine, 21(1), 93-108.

McElroy, S. L., Pope, H. G., Jr., Hudson, J. I., Keck, P. E., Jr., \& White, K. L. (1991). Kleptomania: A report of 20 cases. American Journal of Psychiatry, 148(5), 652-657. doi:10.1176/ajp.148.5.652

Mitchell, J. E., Fletcher, L., Gibeau, L., Pyle, R. L., \& Eckert, E. (1992). Shoplifting in bulimia nervosa. Comprehensive Psychiatry, 33(5), 342-345.

Regan, P., Cachelin, F. M., \& Minnick, A. M. (2017). Initial treatment seeking from professional health care providers for eating disorders: A review and synthesis of potential barriers to and facilitators of "first contact. International Journal of Eating Disorders, 50(3), 190209. doi:10.1002/eat.22683

Rhee, S. H., \& Waldman, I. D. (2002). Genetic and environmental influences on antisocial behavior: A meta-analysis of twin and adoption studies. Psychological Bulletin, 128(3), 490-529.

Smink, F. R., van Hoeken, D., \& Hoek, H. W. (2013). Epidemiology, course, and outcome of eating disorders. Current Opinion in Psychiatry, 26(6), 543-548. doi:10.1097/YCO.0b013e328365a24f

Swedish National Board of Health and Welfare. (1987). Klassifikation av Sjukdomar. Retrieved from https://www.socialstyrelsen.se/ klassificeringochkoder/Documents/KS87-P.pdf

Vandereycken, W., \& van Houdenhove, V. D. (1996). Stealing behavior in eating disorders: Characteristics and associated psychopathology. Comprehensive Psychiatry, 37(5), 316-321.

Swedish National Council for Crime Prevention. (2008). Brottsoffers bengägenhet att anmäla brott, rapport 2008:12. Retrieved from https://www.bra.se/download/18.cba82f7130f475a2f180006127/ 1371914721740/2008_12_brottsoffers_benagenhet_polisanmala_brott. pdf.

Vinkers, D. J., de Beurs, E., Barendregt, M., Rinne, T., \& Hoek, H. W. (2011). The relationship between mental disorders and different types of crime. Criminal Behaviour and Mental Health, 21(5), 307-320. doi:10.1002/cbm.819

Whiteford, H. A., Degenhardt, L., Rehm, J., Baxter, A. J., Ferrari, A. J., Erskine, H. E., ... Vos, T. (2013). Global burden of disease attributable to mental and substance use disorders: Findings from the Global Burden of Disease Study 2010. Lancet, 382(9904), 1575-1586. doi: 10.1016/S0140-6736(13)61611-6 
Wildes, J. E., \& Marcus, M. D. (2013). Alternative methods of classifying eating disorders: Models incorporating comorbid psychopathology and associated features. Clinical Psychological Review, 33(3), 383-394. doi:10.1016/j.cpr.2013.01.006

Yao, S., Kuja-Halkola, R., Thornton, L. M., Runfola, C. D., D'onofrio, B. M., Almqvist, C., ... Bulik, C. M. (2016). Familial liability for eating disorders and suicide attempts: Evidence from a population registry in Sweden. JAMA Psychiatry, 73(3), 284-291. doi:10.1001/ jamapsychiatry.2015.2737

Yilmaz, Z., Hardaway, J. A., \& Bulik, C. M. (2015). Genetics and epigenetics of eating disorders. Advances in Genomics and Genetics, 5, 131-150. doi:10.2147/AGG.S55776

\section{SUPPORTING INFORMATION}

Additional Supporting Information may be found online in the supporting information tab for this article.

How to cite this article: Yao S, Kuja-Halkola R, Thornton LM, et al. Risk of being convicted of theft and other crimes in anorexia nervosa and bulimia nervosa: A prospective cohort study in a Swedish female population. Int J Eat Disord. 2017;50:10951103. https://doi.org/10.1002/eat.22743 